\title{
A synchrotron self-Compton model with low-energy electron cut-off for the blazar S5 $0716+714$
}

\author{
O. Tsang and J. G. Kirk
}

\author{
Max-Planck-Institut-für Kernphysik, Saupfercheckweg 1, 69117 Heidelberg, Germany \\ e-mail: olivia.tsang@mpi-hd.mpg.de
}

Received 28 May 2007 / Accepted 6 September 2007

ABSTRACT

\begin{abstract}
Context. In a self-absorbed synchrotron source with power-law electrons, rapid inverse Compton cooling sets in when the brightness temperature of the source reaches $T_{\mathrm{B}} \sim 10^{12} \mathrm{~K}$. However, brightness temperatures inferred from observations of intra-day variable sources (IDV) are well above the "Compton catastrophe" limit. This can be understood if the underlying electron distribution cuts off at low energy.

Aims. We examine the compatibility of the synchrotron and inverse Compton emission of an electron distribution with low-energy cut-off with that of IDV sources, using the observed spectral energy distribution of S5 0716+714 as an example.

Methods. We compute the synchrotron self-Compton (SSC) spectrum of monoenergetic electrons and compare it to the observed spectral energy distribution (SED) of S5 0716+714. The hard radio spectrum is well-fitted by this model, and the optical data can be accommodated by a power-law extension to the electron spectrum. We therefore examine the scenario of an injection of electrons, which is a double power law in energy, with a hard low-energy component that does not contribute to the synchrotron opacity.

Results. We show that the double power-law injection model is in good agreement with the observed SED of S5 0716+714. For intrinsic variability, we find that a Doppler factor of $\mathcal{D} \geq 30$ can explain the observed SED provided that low-frequency $(<32 \mathrm{GHz})$ emission originates from a larger region than the higher-frequency emission. To fit the entire spectrum, $\mathcal{D} \geq 65$ is needed. We find the constraint imposed by induced Compton scattering at high $T_{\mathrm{B}}$ is insignificant in our model.

Conclusions. We confirm that electron distribution with a low-energy cut-off can explain the high brightness temperature in compact radio sources. We show that synchrotron spectrum from such distributions naturally accounts for the observed hard radio continuum with a softer optical component, without the need for an inhomogeneous source. The required low energy electron distribution is compatible with a relativistic Maxwellian.
\end{abstract}

Key words. galaxies: active - galaxies: high-redshift - galaxies: jets - BL Lacertae objects: individual: S5 0716+714

\section{Introduction}

Observations of many extra-galactic radio sources have found rapid flux variations at radio frequency (e.g. Kedziora-Chudczer et al. 2001), some of which fluctuate over a time scale of a day or less. They are referred to as intra-day variable sources (IDV). The variability time scale is often used to constrain the size of the source based on causality arguments. Using this constraint, one can derive a variability brightness temperature (Wagner \& Witzel 1995)

$T_{\text {var }}=4.5 \times 10{ }^{10} F_{v}\left(\frac{\lambda d_{\mathrm{L}}}{t_{\mathrm{obs}}(1+z)}\right)^{2} \mathrm{~K}$

where the flux density $F_{v}$, wavelength $\lambda$, luminosity distant $d_{\mathrm{L}}$, and observed variability time scale $t_{\mathrm{obs}}$ are measured in $\mathrm{Jy}, \mathrm{cm}$, Mpc, and days, respectively.

The high radio flux frequently measured in IDV sources implies an extremely high brightness temperature, often many orders of magnitude above $10^{12} \mathrm{~K}$. Kellermann \& Pauliny-Toth (1969) have shown that, assuming the electron distribution follows a single power law, the luminosity of the inverse Compton scattered photons exceeds that of the synchrotron photons when the brightness temperature of the source reaches $\sim 10^{12} \mathrm{~K}$. Above this threshold, rapid cooling of the relativistic electrons due to inverse Compton scattering - the "Compton catastrophe" - forbids a further increase in the brightness temperature (see e.g.
Kellermann 2002, for a recent review of the brightness temperature problem). The limiting value is even lower, $T_{\mathrm{B}}<10^{11} \mathrm{~K}$, if the magnetic field and particle energy density of the source is driven towards equipartition (Readhead 1994). The observed variability in some sources can be interpreted as the result of extrinsic effects, which, at first sight, relaxes the size constraint. For example, the flux variations of PKS 1519-273 and PKS 0405-385 are convincingly identified as interstellar scintillation. Nevertheless, all realistic models of the scintillation mechanism impose a new constraint on the size and require a brightness temperature of $T_{\mathrm{B}}>10^{13} \mathrm{~K}$ in some cases (Macquart et al. 2000; Rickett et al. 2002), far exceeding the limit imposed by the Compton catastrophe.

A prevalent feature associated with IDV sources is a flat or inverted spectrum ( $\alpha \leq 0$, with flux $F_{v} \propto v^{-\alpha}$ ) at radio-millimeter wavelengths (e.g., Gear et al. 1994; Kedziora-Chudczer et al. 2001). Optically thick synchrotron emission from power-law electrons rises as $v^{5 / 2}$, too fast to account for the observed spectra. Optically thin synchrotron emission in the scope of the conventional interpretation of the synchrotron theory has a flux $F_{v} \propto v^{-(s-1) / 2}$, where $s$ is the power-law index of the electrons $\left(\mathrm{d} N_{\mathrm{e}} / \mathrm{d} \gamma \propto \gamma^{-s}\right)$. If $\alpha=$ $(s-1) / 2 \leq 0$, the number density of electrons diverges towards high $\gamma$. Imposing a high-energy cut-off in the electron spectrum avoids the divergence and may account for the commonly observed spectral steepening at optical frequencies, but 
Marscher (1977) showed that electron spectra with $s \leq 1$ would result in a high flux between infrared and optical frequencies that is not supported by observations. The most common interpretation of the flat or inverted spectra is, therefore, a superposition of many synchrotron spectra within an inhomogeneous source (e.g. de Bruyn 1976; Marscher 1977; Blandford \& Konigl 1979).

In Kirk \& Tsang (2006), we discussed a synchrotron selfCompton model in which the electron distribution is monoenergetic. The lack of low-energy electrons enables more $\mathrm{GHz}$ photons to emerge from the source, allowing a higher brightness temperature to be observed without initiating catastrophic cooling. We found that a temperature of up to $T_{\mathrm{B}} \sim 10^{14} \mathrm{~K}$ at $\mathrm{GHz}$ frequencies is possible with only a moderate Doppler boosting factor of $\sim 10$. In Tsang \& Kirk (2007), we discussed the parameters of the monoenergetic model and showed that the assumption of equipartition of energy in the source does not prevent the Compton catastrophe. We also showed that an injection of highly relativistic electrons or strong acceleration in the source cannot produce temperatures much higher than our limit due to copious electron-positron pair production.

In this paper, we examine the spectral properties of synchrotron emission from monoenergetic electrons and from an electron distribution that is a double power law in energy, by comparing the model spectra with the observations of S5 $0716+714$, a BL Lac object that is one of the brightest known IDV sources, as well as a gamma-ray blazar (Hartman et al. 1999). In doing so, we assume that the dominant targets for inverse Compton scattering are produced within the source (SSC model). The emission from gamma-ray blazars can also be interpreted in the context of models in which the target photons are created externally (EC model), for example in the broad line region, the accretion disk, or a molecular torus (Sokolov \& Marscher 2005). However, in many sources there is no observational evidence of a significant external photon source. This is the case for S5 0716+714, where, despite much effort over the past three decades, no emission lines have been detected (e.g., Bychkova et al. 2006). Furthermore, XMM-Newton observations of S5 $0716+714$ in 2004 analysed by Ferrero et al. (2006) and Foschini et al. (2006) show two spectral components in the $0.5-10 \mathrm{keV}$ band, whose variability properties appear to favour the SSC interpretation. The recent extensive simultaneous observations of this object from radio to optical frequencies by Ostorero et al. (2006), together with INTEGRAL pointings at $\mathrm{GeV} \gamma$-ray energies during the same period, provide the best test for our model.

In the following, we present the computation of the stationary electron distribution and the resulting synchrotron and inverse Compton spectra. The model spectra computed using the monoenergetic electron approximation, as described in Tsang \& Kirk (2007), are presented first. Although adequate for the radio emission, the monoenergetic model cannot reproduce the entire spectrum of S5 $0716+714$. We therefore investigate an electron distribution that is a double power law in energy - a hard lowenergy part that softens to a high-energy tail above a characteristic energy. In this way, the inverted optically thin radio emission is retained and complemented by nonthermal synchrotron emission from the high energy tail. In Sect. 2, we briefly describe these injection models. The resulting stationary electron distribution is calculated in Sect. 3 and used for the computation of the synchrotron and inverse Compton spectra. In Sect. 4, we compare the predictions of these models with the observed spectral energy distribution (SED) of the source to S5 0716+714. Our findings and some limitations of our approach are discussed in Sect. 5 and our conclusions presented in Sect. 6.

\section{The model}

The homogeneous monoenergetic model discussed previously (Kirk \& Tsang 2006; Tsang \& Kirk 2007) can be completely characterised by the Doppler boosting factor $\mathcal{D}=$ $1 /[\Gamma(1-\beta \cos \vartheta)](c \beta$ is the source speed with respect to the rest frame of the host galaxy, $\vartheta$ the angle between the velocity and the line of sight, and $\left.\Gamma=\left(1-\beta^{2}\right)^{-1 / 2}\right)$, the redshift of the host galaxy $z$, and four source parameters, the electron number density $N_{\mathrm{e}}$, the magnetic field strength $B$, the linear size of the source $R$, and the electron Lorentz factor measured in the rest frame of the source $\gamma$. For the purpose of comparison with observations, these can be transformed into a different set of parameters. Details of the transformation can be found in Kirk \& Tsang (2006), in which $N_{\mathrm{e}}, B$, and $\gamma$ are replaced by the characteristic frequency of synchrotron emission, $v_{\mathrm{s}}=\gamma^{2} v_{0}$, where $v_{0}=3 e B /(4 \pi m c)$, the Comptonisation parameter $\xi$, which is the ratio of the luminosity of each successive generation of inverse Compton scattered photons to the luminosity of the previous generation: $\xi=4 \gamma^{2} \tau_{\mathrm{T}} / 3$, (where $\tau_{\mathrm{T}}=N_{\mathrm{e}} R \sigma_{\mathrm{T}}$ is the Thomson optical depth), and the optical depth $\tau_{\mathrm{s}}$ to synchrotron selfabsorption at the observing frequency. The size of the source, $R$, can be constrained, for example, by applying causality arguments to the variation time, $\Delta t$, of the source: $R<c \Delta t \mathcal{D} /(1+z)$.

We present in Sect. 4 the model spectra from monoenergetic electrons that show good agreement with the observations of S5 $0716+714$ at radio frequencies. The optical data can be fitted by this model if a high-energy power-law "tail" is added. To do this, we consider an injection spectrum of the form $Q(\gamma) \propto\left(\gamma / \gamma_{\mathrm{p}}\right)^{-s}$ for $\gamma_{\min }<\gamma<\gamma_{\max }$, where the power-law index $s$ equals $s_{1}$ for $\gamma<\gamma_{\mathrm{p}}$, and $s_{2}$ for $\gamma>\gamma_{\mathrm{p}}$ (Fig. 1). The electron number density at a given time is proportional to $\gamma_{\max }^{1-s}$ for $s<1, \propto \gamma_{\min }^{1-s}$ for $s>1$, and $\propto \ln \gamma_{\max }$ for $s=1$. In the highenergy branch of the injection spectrum, for $\gamma>\gamma_{\mathrm{p}}$, we require that $s_{2}>1$, so that electron number density congregates towards $\gamma_{\mathrm{p}}$. In the low-energy branch, $\gamma<\gamma_{\mathrm{p}}$, the electrons congregate at $\gamma_{\mathrm{p}}$ if $s_{1}<1$. But we also require that the opacity to synchrotron self-absorption is dominated by electrons with $\gamma=\gamma_{\mathrm{p}}$, which is achieved by demanding $s_{1}<1 / 3$. Under the conditions $s_{1}<1 / 3$ and $s_{2}>1$, the low-frequency synchrotron spectrum is wellapproximated by that of monoenergetic electrons with Lorentz factor $\gamma_{\mathrm{p}}$.

The electron injection spectrum cuts off at $\gamma_{\min }$ towards low energy and at $\gamma_{\max }$ towards high energy. The exact value of $\gamma_{\min }$ is unimportant, since, as explained above, synchrotron emission and opacity are dominated by electrons with $\gamma=\gamma_{\mathrm{p}}$ in the low-energy part of the injection spectrum, where $\gamma_{\max }$ determines the high frequency cut-off in the synchrotron spectrum, at $v_{\max }=\gamma_{\max }^{2} v_{0}$, and the highest photon energy achievable through inverse Compton scattering in the Klein-Nishina limit, which equals $\gamma_{\max } m c^{2}$.

To summarise, the injection spectrum has the form

$Q(\gamma)=Q_{0} \begin{cases}\left(\gamma / \gamma_{\mathrm{p}}\right)^{-s_{1}}, & \gamma_{\min } \leq \gamma<\gamma_{\mathrm{p}} \\ \left(\gamma / \gamma_{\mathrm{p}}\right)^{-s_{2}}, & \gamma_{\mathrm{p}} \leq \gamma<\gamma_{\max }\end{cases}$

where $Q_{0}$ is the electron injection rate per unit volume per unit $\gamma$ at $\gamma=\gamma_{\mathrm{p}}$.

\section{Stationary solution}

The shape of the synchrotron spectrum is determined by the stationary electron-energy distribution. Electrons injected into the 
$\log _{10} Q(\gamma) / \log _{10} n(\gamma)$

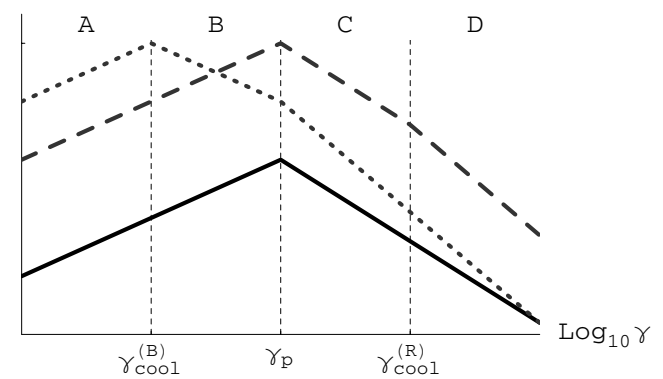

Fig. 1. Schematic representation of the electron injection spectrum and the stationary differential number density as a function of $\gamma$. The heights of the spectra have been adjusted for easy comparison and are not to scale. The solid line shows the double power-law injection spectrum with power-law index $s_{1}$ for $\gamma<\gamma_{\mathrm{p}}$, and $s_{2}$ for $\gamma>\gamma_{\mathrm{p}}$. The dashed line shows the case where $\gamma_{\text {cool }}=\gamma_{\text {cool }}^{(\mathrm{R})}>\gamma_{\mathrm{p}}$. The differential electron number density $n_{\mathrm{e}} \propto \gamma^{-s_{1}}$ in regions A and $\mathrm{B}, n_{\mathrm{e}} \propto \gamma^{-s_{2}}$ in region $\mathrm{C}$, and $n_{\mathrm{e}} \propto \gamma^{-\left(s_{2}+1\right)}$ in region D. The dotted line shows the case where $\gamma_{\text {cool }}=\gamma_{\text {cool }}^{(\mathrm{B})}<\gamma_{0}$, with $n_{\mathrm{e}} \propto \gamma^{-s_{1}}$ in region $\mathrm{A}, n_{\mathrm{e}} \propto \gamma^{-2}$ in region $\mathrm{B}$, and $n_{\mathrm{e}} \propto \gamma^{-\left(s_{2}+1\right)}$ in regions $\mathrm{C}$ and $\mathrm{D}$.

source according to Eq. (2) are subject to radiative cooling while in the source and evacuate this zone on a time-scale close to the light crossing time, $t_{\mathrm{esc}} \sim R / c$. The evolution of the electron spectrum is governed by the kinetic equation (Kardashev 1962):

$\frac{\partial n_{\mathrm{e}}}{\partial t}=Q_{0}\left(\frac{\gamma}{\gamma_{\mathrm{p}}}\right)^{-s}-\frac{\partial}{\partial \gamma}\left(n_{\mathrm{e}} \dot{\gamma}_{\text {total }}\right)-\frac{n_{\mathrm{e}}}{t_{\mathrm{esc}}}$

where, for simplicity, we denote the differential electron number density $\left(\mathrm{d} N_{\mathrm{e}} / \mathrm{d} \gamma\right)$ by $n_{\mathrm{e}}$. The second term on the right hand side of Eq. (3) is the rate of change of the electron Lorentz factor due to radiative losses. This term is the sum of the rates for synchrotron losses and for losses from inverse Compton scattering:

$\dot{\gamma}_{\text {total }}=\dot{\gamma}_{\mathrm{s}}+\dot{\gamma}_{\mathrm{IC}}$

where

$\dot{\gamma}_{\mathrm{s}}=\frac{4 \sigma_{\mathrm{T}} U_{\mathrm{B}}}{3 m c} \gamma^{2}$.

The third term is the rate at which electrons escape from the source.

In the stationary state, Eq. (3) can be solved analytically:

$n_{\mathrm{e}}(\gamma)=\frac{1}{f_{\mathrm{I}}(\gamma)} \int^{\gamma} \frac{Q\left(\gamma^{\prime \prime}\right)}{\gamma^{\prime \prime}{ }_{\text {total }}} f_{\mathrm{I}}\left(\gamma^{\prime \prime}\right) \mathrm{d} \gamma^{\prime \prime}$

with the integrating factor

$f_{\mathrm{I}}(\gamma)=\dot{\gamma} \exp \left[-\int^{\gamma}\left({\dot{\gamma^{\prime}}}_{\text {total }} t_{\mathrm{esc}}\right)^{-1} \mathrm{~d} \gamma^{\prime}\right]$.

However, Eq. (3) is only a rough description of a source, for example, because of the crude treatment of particle escape involved in setting $t_{\mathrm{esc}}=R / c$. Therefore, rather than use Eq. (6), we choose to use an approximate solution that more clearly demonstrates the effects that cooling and the evacuation of electrons from the emission region have on the electron energy distribution.

We first identify the Lorentz factor, $\gamma_{\text {cool }}$, which determines the electron energy at which radiative cooling dominates losses due to particles escaping the emission region:

$\left.\frac{\dot{\gamma}_{\text {total }}}{\gamma}\right|_{\gamma=\gamma_{\text {cool }}}=\frac{1}{t_{\text {esc }}}$.
In principle, $\gamma_{\text {cool }}$ can be evaluated only if the entire electron distribution is already known, since $\dot{\gamma}_{\mathrm{IC}}$ depends on the spectrum and intensity of emitted radiation. However, in practise, a simple iterative scheme enables it to be found rapidly in all the cases we have computed. Assuming it is known, solutions of Eq. (3) that are valid in the limits $\gamma \ll \gamma_{\text {cool }}$ and $\gamma \gg \gamma_{\text {cool }}$ are easily found. In the first case, cooling is unimportant, and it immediately follows that

$n_{\mathrm{e}}=t_{\mathrm{esc}} Q_{0}\left(\gamma / \gamma_{\mathrm{p}}\right)^{-s} \quad$ for $\gamma \ll \gamma_{\text {cool }}$.

In the second, escape is unimportant, and the appropriate solution is found by integrating the kinetic equation once:

$n_{\mathrm{e}}=\dot{\gamma}^{-1} \int_{\gamma}^{\infty} Q_{0}\left(\gamma^{\prime} / \gamma_{\mathrm{p}}\right)^{-s} \mathrm{~d} \gamma^{\prime} \quad$ for $\gamma \gg \gamma_{\mathrm{cool}}$.

These solutions intersect close to the point $\gamma=\gamma_{\text {cool }}$. Our approximation consists in adopting the solution without cooling given in Eq. (9) for all Lorentz factors below the intersection point and the solution without escape given in Eq. (10) for all Lorentz factors above the intersection point.

In addition, we assume and verify a posteriori (see Sect. 5) that $\dot{\gamma}_{\mathrm{IC}}$ can be approximated by the expression for inverse Compton scattering of the synchrotron photons in the Thomson regime:

$\dot{\gamma}_{\mathrm{IC}}=\frac{4 \sigma_{T} U_{\mathrm{s}}}{3 m c} \gamma^{2}$

where $U_{\mathrm{s}}$ is the energy density of synchrotron photons in the source. In this case, $\dot{\gamma}_{\text {total }}=\gamma^{2} /\left(\gamma_{\text {cool }} t_{\text {esc }}\right)$, and our approximate solution is

$n_{\mathrm{e}}= \begin{cases}t_{\mathrm{esc}} Q(\gamma) & \gamma<a \gamma_{\mathrm{cool}} \\ t_{\mathrm{esc}} \gamma_{\mathrm{cool}} \gamma^{-2} \int_{\gamma}^{\infty} \mathrm{d} \gamma^{\prime} Q\left(\gamma^{\prime}\right) & a \gamma_{\mathrm{cool}} \leq \gamma\end{cases}$

where $a(\sim 1)$ is determined by requiring the solution (but not its first derivative) to be continuous.

The Lorentz factors $\gamma_{\text {cool }}$ and $\gamma_{\mathrm{p}}$ give rise to breaks in $n_{\mathrm{e}}$, which correspond to the breaks in the synchrotron spectrum at $v_{\mathrm{p}}=\gamma_{\mathrm{p}}^{2} v_{0}$ and $v_{\text {cool }}=\gamma_{\text {cool }}^{2} v_{0}$. Notice that, if $s<1$ (as in the injection spectrum below $\gamma_{\mathrm{p}}$ ), $n_{\mathrm{e}}$ is approximately proportional to $\dot{\gamma}^{-1} \propto \gamma^{-2}$, whereas if $s>1$ (as in the injection spectrum above $\left.\gamma_{\mathrm{p}}\right), n_{\mathrm{e}}$ is approximately $\propto \gamma^{-(s+1)}$.

Two types of stationary spectra result from Eq. (12), depending on whether the peak of the injection spectrum, $\gamma_{\mathrm{p}}$, is below or above $\gamma_{\text {cool }}$. Figure 1 shows the injection spectrum as a solid line, the stationary spectra where $\gamma_{\mathrm{p}}>\gamma_{\mathrm{cool}}$ as a dotted line and where $\gamma_{\mathrm{p}}<\gamma_{\mathrm{cool}}$ as a dashed line. When electrons are predominantly removed from a certain energy range by leaving the source $\left(t_{\text {esc }}<t_{\text {cool }}\right)$, the spectrum retains its original shape, $n_{\mathrm{e}} \propto \gamma^{-s}$, since $t_{\mathrm{esc}}$ is independent of particle energy. On the other hand, when synchrotron losses dominates, such that $t_{\text {esc }}>t_{\text {cool }}$, the stationary solution is $n_{\mathrm{e}} \propto \gamma^{-2}$ for $\gamma<\gamma_{\mathrm{p}}$, and $n_{\mathrm{e}} \propto \gamma^{-(s+1)}$ for $\gamma>\gamma_{\mathrm{p}}$. For the computation of the low frequency synchrotron emission, the distribution can be approximated by a monoenergetic one at $\gamma_{\mathrm{cool}}$ in the first case and $\gamma_{\mathrm{p}}$ in the second.

The iterative procedure used to find $\gamma_{\text {cool }}$ is as follows: the loss rate is defined as

$\dot{\gamma}_{\text {total }}=\frac{4 \sigma_{T} U_{\mathrm{B}}(1+\delta)}{3 m c} \gamma$.

Then, starting with $\delta=0, \gamma_{\text {cool }}$ is evaluated from Eq. (8) and, using the electron distribution given by (12), $U_{\mathrm{s}}$ is evaluated as 
described in Sect. 3.1. The value of $\delta$ is readjusted to $\delta=U_{\mathrm{s}} / U_{\mathrm{B}}$ and the cycle repeated until successive values differ by less than $1 \%$. In the examples discussed in this paper, convergence was achieved after two iterations. Because the change in $\gamma_{\text {cool }}$ between iterations was only roughly a factor of 2 , the final emission spectrum was close to that found using $\delta=0$.

\subsection{Synchrotron and inverse Compton emission}

The synchrotron specific intensity, following straightforwardly from the radiative transport equation, is

$I_{v}^{(\mathrm{S})}=S_{v}\left[1-\exp \left(-\tau_{\mathrm{S}}\right)\right]$

where the optical depth to synchrotron radiation is $\tau_{\mathrm{s}}=$ $\alpha_{v} \cdot R$, and $\alpha_{v}$ is the absorption coefficient (e.g., Longair 1992, Chap. 18)

$$
\begin{aligned}
\alpha_{v}= & -\frac{3 \sqrt{3}}{16} \frac{\sigma_{\mathrm{T}}}{\alpha_{\mathrm{f}}} \frac{m c^{2}}{h v} \frac{\nu_{\mathrm{L}} \sin \phi}{v} \\
& \times \int_{\gamma_{\min }}^{\gamma_{\max }} \gamma^{2} F(x) \frac{\mathrm{d}}{\mathrm{d} \gamma}\left(\frac{n_{\mathrm{e}}(\gamma)}{\gamma^{2}}\right) \mathrm{d} \gamma
\end{aligned}
$$

where $\alpha_{\mathrm{f}}$ is the fine structure constant, $\phi$ the angle between the magnetic field and the direction of the emitted radiation, $x=v /\left(\gamma^{2} v_{0}\right), F(x)=x \int_{x}^{\infty} K_{5 / 3}(t) \mathrm{d} t$, and $K_{5 / 3}$ is the modified Bessel function of order $5 / 3$. The source function $S_{v}$ is

$S_{v}=-2 m v^{2} \frac{\int_{\gamma_{\min }}^{\gamma_{\max }} F(x) n_{\mathrm{e}}(\gamma) \mathrm{d} \gamma}{\int_{\gamma_{\min }}^{\gamma_{\max }} \gamma^{2} F(x) \frac{\mathrm{d}}{\mathrm{d} \gamma}\left(\frac{n_{\mathrm{e}}(\gamma)}{\gamma^{2}}\right) \mathrm{d} \gamma}$.

In the monoenergetic approximation, the source function simplifies to

$S_{v}=m v_{0}^{2} \frac{\gamma^{5} F(x)}{K_{5 / 3}(x)}$.

Equation (14) is integrated over frequency and angle to give the energy density of synchrotron photons in the source

$U_{\mathrm{s}}=\frac{4 \pi}{3 c} \zeta \int_{0}^{\infty} I_{\nu} \mathrm{d} \nu$

where $\zeta$ is a geometrical factor that is shown in Tsang \& Kirk (2007) to be $\zeta=2 / 3$.

The synchrotron photons are repeatedly scattered by the energetic electrons to higher energies. Denoting by $i$ the number of times a photon is scattered, the rate of scattering the $(i-1)$ th generation of photons into the frequency interval $\mathrm{d} v_{i}$ by a single electron (see e.g., Georganopoulos et al. 2001, Eq. (4)) is

$\left(\frac{\mathrm{d} n_{\mathrm{ph}}}{\mathrm{d} t \mathrm{~d} v_{i}}\right)_{\mathrm{sp}}=\frac{3 \sigma_{\mathrm{T}} c}{4 v_{i-1} \gamma^{2}} f(y) N_{v_{i-1}}$

where

$N_{v_{i-1}}=\frac{4 \pi}{c} \frac{\zeta I_{v_{i-1}}}{h v_{i-1}}$

is the number density of the target photons, and $I_{v_{i-1}}$ the specific intensity of the $(i-1)^{\text {th }}$ generation of photons. The first generation of scattered photons is produced directly from the synchrotron photons: $i=1, I_{v_{0}}=I_{v}^{(\mathrm{S})}$. Rybicki \& Lightman (1979, Chap. 7) assumed that scattering in the Thomson regime is isotropic in the rest frame of the electron, and obtained $f(y) \approx f_{\text {iso }}(y)=2(1-y) / 3$. Here, we include the Klein-Nishina effects (e.g., Georganopoulos et al. 2001), in which case

$$
\begin{aligned}
f(y)= & {\left[2 y \ln y+y+1-2 y^{2}+\frac{\left(4 \epsilon_{i-1} \gamma y\right)^{2}(1-y)}{2\left(1+4 \epsilon_{i-1} \gamma y\right)}\right] } \\
& \times P\left(1 / 4 \gamma^{2}, 1, y\right), \\
y= & \frac{\epsilon_{i}}{4 \epsilon_{i-1} \gamma^{2}\left(1-\epsilon_{i} / \gamma\right)}
\end{aligned}
$$

where $\epsilon_{i-1}$ and $\epsilon_{i}$ are the energy of the target photons and scattered photon, respectively, in units of $m c^{2}$, and $P\left(1 / 4 \gamma^{2}, 1, y\right)=1$ for $1 / 4 \gamma^{2} \leq y \leq 1$, and zero otherwise.

Assuming a spherical source, the rate of scattering photons with energy $h v_{i-1}$ to energy $h v_{i}$, in the observer's frame, from a homogeneous distribution of electrons with differential number density $n_{\mathrm{e}}$ can be found by integrating over the electron energy distribution,

$\left(\frac{\mathrm{d} n_{\mathrm{ph}}}{\mathrm{d} t \mathrm{~d} v_{i}}\right)=\frac{4 \pi}{3}\left(\frac{R}{2}\right)^{3} \int_{0}^{\infty} \mathrm{d} \gamma n_{\mathrm{e}}\left(\frac{\mathrm{d} n_{\mathrm{ph}}}{\mathrm{d} t \mathrm{~d} v_{i}}\right)_{\mathrm{sp}}$.

Note that $R$ (the linear size of the source) is divided by 2 to obtain the source radius.

The specific intensity of the $i$ th generation photons is then the scattering rate of the electron distribution in Eq. (23) integrated over all target photon frequency,

$$
\begin{aligned}
I_{v_{i}}^{(\mathrm{C})} & =\left(\frac{\mathrm{d} E}{\mathrm{~d} t \mathrm{~d} v_{i} \mathrm{~d} r^{2} \mathrm{~d} \Omega}\right) \\
& =\int_{0}^{\infty} \mathrm{d} v_{i-1}\left(\frac{\mathrm{d} n_{\mathrm{ph}}}{\mathrm{d} t \mathrm{~d} v_{i}}\right) \frac{h v_{i}}{4 \pi(R / 2)^{2}},
\end{aligned}
$$

and for a general electron distribution $n_{\mathrm{e}}$, it can be written as

$I_{v_{i}}^{(\mathrm{C})}=\frac{\pi}{3} R \sigma_{\mathrm{T}} v_{i} \int_{0}^{\infty} \frac{\mathrm{d} \gamma}{\gamma^{2}} n_{\mathrm{e}} \int_{0}^{\infty} \frac{\mathrm{d} v_{i-1}}{v_{i-1}^{2}} I_{v_{i-1}} f(y)$.

For a monoenergetic electron distribution, Eq. (25) can be simplified to

$I_{v_{i}}^{(\mathrm{C})}=\frac{\pi}{3} \tau_{\mathrm{T}} \frac{v_{i}}{\gamma^{2}} \int_{0}^{\infty} \frac{\mathrm{d} v_{i-1}}{v_{i-1}^{2}} I_{v_{i-1}} f(y)$.

Equations (26) and (25) are integrated numerically for monoenergetic electrons and for an electron distribution given by Eq. (12).

\section{The BL Lac object S5 $0716+714$}

Observations of S5 0716+714 have shown that the source exhibits intra-day variability in the radio and optical bands (e.g. Ghisellini et al. 1997; Raiteri et al. 2003). Correlation between radio (at $5 \mathrm{GHz}$ ) and optical (at $650 \mathrm{~nm}$ ) variability suggest that scintillation, a process that is not effective at high radio and optical frequencies, does not play a large part in the observed variability (Quirrenbach et al. 1991; Wagner 2001). More recent multi-frequency studies of S5 0716+714 (e.g. Ostorero et al. 2006; Agudo et al. 2006) have obtained simultaneous measurements from radio to optical frequencies during the INTEGRAL pointing period, and the non-detection of the source by INTEGRAL has provided upper limits at X-ray frequencies. Flux variations were detected at 32 and $37 \mathrm{GHz}$ over a period of $\Delta t=4.1$ days (in November 2003) when the two-frequency measurements overlap. Since interstellar scintillation is ineffective at these frequencies, Ostorero et al. (2006) conclude that 
Table 1. Model parameters corresponding to Figs. 2 and 3.

\begin{tabular}{lllll}
\hline \hline & Mono (dashed) & Mono (solid) & Power-law (dashed) & Power-law (solid) \\
\hline Primary parameters & & & & \\
$z$ & 0.3 & 0.3 & 0.3 & 0.3 \\
$\Delta t$ (days) & 4.1 & 4.1 & 4.1 & 4.1 \\
$\mathcal{D}$ & 55 & 55 & 30 & 65 \\
$v_{\mathrm{p}}(\mathrm{Hz})$ & $5.5 \times 10^{13}$ & $3.9 \times 10^{11}$ & $2.0 \times 10^{11}$ & $2.7 \times 10^{11}$ \\
$v_{\text {cool }}(\mathrm{Hz})$ & $1.74 \times 10^{12}$ & $1.17 \times 10^{19}$ & $2.07 \times 10^{16}$ & $2.14 \times 10^{19}$ \\
$v_{\max }(\mathrm{Hz})$ & $\ldots$ & $\ldots$ & $1.0 \times 10^{18}$ & $1.5 \times 10^{15}$ \\
$\xi$ & $10^{-2.5}$ & $10^{-0.75}$ & $\ldots$ & $\ldots$ \\
\hline Secondary parameters & & & & \\
$\xi$ & $\ldots$ & $\ldots$ & 0.92 & 0.86 \\
$R(\mathrm{pc})$ & 0.15 & 0.15 & 0.08 & 0.17 \\
$\theta_{\mathrm{d}}(\mu \mathrm{as})$ & 32.5 & 32.5 & 17.7 & 38.4 \\
$\gamma_{\mathrm{p}}$ & 691 & 800 & 244 & 696 \\
$\gamma_{\text {cool }}$ & 123 & $4.39 \times 10^{6}$ & $7.85 \times 10^{4}$ & $6.19 \times 10^{6}$ \\
$\gamma_{\text {max }}$ & $\ldots$ & $\ldots$ & $5.45 \times 10^{5}$ & $5.19 \times 10^{4}$ \\
$\left.N_{\mathrm{e}}(\mathrm{cm})^{-3}\right)$ & 0.02 & 0.70 & 3.15 & 0.32 \\
$B(\mathrm{mG})$ & 648 & 3.43 & 34.7 & 2.65 \\
$\delta$ & $\ldots$ & $\ldots$ & 0.97 & 0.93 \\
$U_{\mathrm{B}} / U_{\mathrm{par}}$ & $1.8 \times 10^{3}$ & $1.0 \times 10^{-3}$ & 0.04 & $2.4 \times 10^{-4}$ \\
$T_{\mathrm{B}}(\mathrm{K})$ & $3.7 \times 10^{12}$ & $3.9 \times 10^{12}$ & $1.4 \times 10^{13}$ & $1 \times 10^{-7}$ \\
$\tau_{\mathrm{T}}$ & $6 \times 10^{-9}$ & $2 \times 10^{-7}$ & $5 \times 10^{-7}$ & $4 \times 10^{43}$ \\
$P_{\text {jet }}(\mathrm{ergs} / \mathrm{s})$ & $1 \times 10^{45}$ & $3 \times 10^{43}$ & $5 \times 10^{42}$ & \\
\hline
\end{tabular}

Note: $\theta_{\mathrm{d}}$ is the angular diameter of the source, $U_{\mathrm{par}}$ the energy density of the particles, and $P_{\text {jet }}$ is the jet power in the rest frame of the host galaxy, as predicted by each model. The compactness of all four models are negligibly small so not included in the discussion.

the variability is intrinsic. Assuming $H_{0}=70 \mathrm{~km} \mathrm{~s}^{-1} \mathrm{Mpc}^{-1}$, with $\Omega_{\lambda}=0.7, \Omega_{\mathrm{M}}=0.3$, and $\Omega_{\mathrm{k}}=0$, and a redshift $z>0.3$ based on the non-detection of a host galaxy (e.g. Wagner et al. 1996), they derive a variability brightness temperature of $T_{\mathrm{var}}>$ $(2.1 \pm 0.1) \times 10^{14} \mathrm{~K}$.

Bach et al. (2005) analysed the data set of VLBI images of 11 jet components of S5 $0716+714$ at $4.9 \mathrm{GHz}, 8.4 \mathrm{GHz}$, $15.3 \mathrm{GHz}$, and $22.2 \mathrm{GHz}$, observed between 1992 and 2001. Assuming that all the jet components move with the same speed along the jet (i.e. all components have the same bulk Lorentz factor), they propose that the observed wide range (from $5.5 c$ to $16.1 c$ ) of apparent component speeds is due to variations in the viewing angle and limit the Lorentz factor and the viewing angle of the VLBI jet to $\Gamma>15$ and $\theta<2^{\circ}$, respectively. Under these conditions, the range of Doppler factors would be $\mathcal{D} \approx 20-30$.

According to Ostorero et al. (2006), observations of S5 $0716+714$ between $5 \mathrm{GHz}$ and $32 \mathrm{GHz}$ can be fitted with spectral indices $\alpha_{5-32}$ of -0.3 and -0.5 at two different epochs. They suggest that the radio observations can be interpreted as optically thick synchrotron emission from an inhomogeneous source, and the spectral break at $v \approx 10^{13} \mathrm{~Hz}$ would correspond to the self-absorption frequency $v_{\text {abs }}$. In the near-infrared to optical band, observations from 2001-2004, reported by Hagen-Thorn et al. (2006) suggest that the spectral energy distribution between the frequencies $v_{\mathrm{K}}=1.38 \times 10^{14} \mathrm{~Hz}$ and $v_{\mathrm{B}}=6.81 \times 10^{14} \mathrm{~Hz}$ can be fitted by the power law $F_{v} \propto v^{-1.12}$.

Here, we apply the two homogeneous models described in Sect. 2: the monoenergetic one, which successfully models the hard radio spectrum with relatively few free parameters, and the one with double power-law injection (and, consequently more free parameters), which also enables the high-energy emission to be modelled. We adopt a value of $z=0.3$ for the red-shift of S5 $0716+714$, and a linear size inferred by the variability time scale of $\Delta t=4.1$ days, so that $R=c \Delta t \mathcal{D} /(1+z)$. The values we find for the free parameters of the models and for several parameters derived from these are given in Table 1 . The spectra predicted by the two models are shown in Figs. 2 and 3, and are discussed separately in the next two sections.

Identifying the creation of our homogeneous source with an event that leads to the ejection of an individual blob observed with VLBI, we estimate the minimum jet power implied by each set of parameters by multiplying the total energy content of the source by the average rate at which blobs are ejected from the core. In the co-moving frame of the source, for monoenergetic electrons, the total energy content is

$E_{\text {blob }}^{\prime}=\left(\frac{B^{2}}{8 \pi}+N_{\mathrm{e}}^{\prime} \gamma m c^{2}\right) R^{\prime 3}$

and, for power-law electrons,

$E_{\text {blob }}^{\prime}=\left(\frac{B^{2}}{8 \pi}+\int_{\gamma_{\min }}^{\gamma_{\max }} n_{\mathrm{e}}^{\prime}(\gamma) \gamma m c^{2} \mathrm{~d} \gamma\right) R^{\prime 3}$

Transforming to the rest frame of the galaxy, $E_{\mathrm{blob}}=\Gamma E_{\mathrm{blob}}^{\prime}$, so that the jet power

$P_{\text {jet }}=E_{\text {blob }}\left(\frac{1}{\Delta t_{\mathrm{ej}}}\right)$

where $\Delta t_{\mathrm{ej}}$ is the average time difference between the ejection of successive blobs. Linear fits of the change in the position of the 11 jet components (Bach et al. 2005) suggest that a new component is ejected from the core every $0.1-1.8$ years. The lower limits to the jet power of each model given in Table 1 were computed using $\Delta t_{\mathrm{ej}}=1.8$ years.

\subsection{Monoenergetic electrons}

In the monoenergetic model, the spectrum is specified by four parameters, $v_{\mathrm{abs}}, v_{\mathrm{p}}, \mathcal{D}$, and $\xi$, as well as $z$ and $\Delta t$ (which are kept fixed for all models). The self-absorption frequency $v_{\mathrm{abs}}$ is determined by the first spectral break at $\sim 4 \mathrm{GHz}$, and $v_{\mathrm{p}}$ corresponds to the spectral cut-off. In Fig. 2, we compare two models 


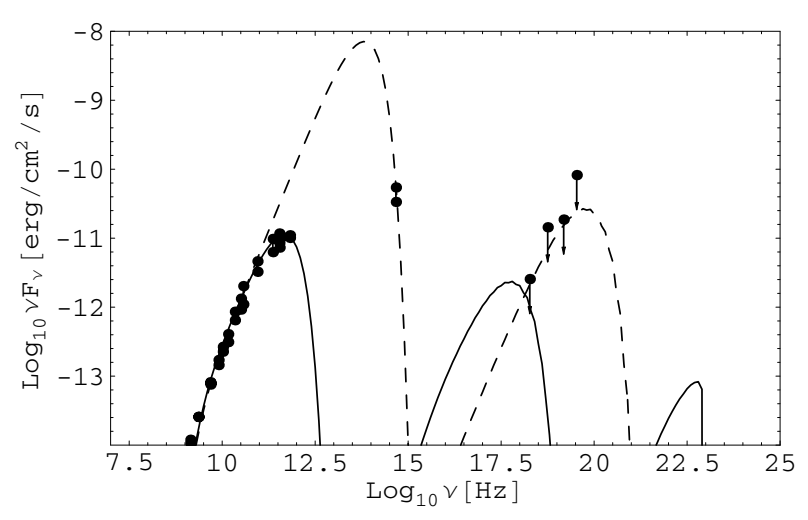

Fig. 2. Spectral energy distribution of S5 $0716+714$. Multi-frequency simultaneous data from Ostorero et al. (2006) are shown as black symbols. Black dots show data points, variation ranges are shown by a vertical bar between two symbols, and downward arrows show upper limits. Values of the parameters are shown in Table 1. The model spectra are computed from a distribution of monoenergetic electrons and are shown with solid and dashed lines. The dashed line shows the strong-magneticfield model where the parameters are chosen such that it goes through the data points at optical frequency, whereas the solid line shows weakmagnetic-field model where the parameters are chosen to mimic the spectral turning at $10^{11.5} \mathrm{~Hz}$. The values of the parameters are shown in Table 1.

in which one has a cut-off at the spectral break at $\sim 10^{11.5} \mathrm{~Hz}$, and the other cuts off just before reaching the optical point. The Doppler factor $\mathcal{D}$ affects the level of the observed flux both by determining the linear size of the source and determining the amount of boosting the flux receives. The Comptonisation parameter $\xi$ determines the ratio of the synchrotron flux to the inverse Compton flux, as well as the value of $\gamma_{\mathrm{p}}$. Therefore, after specifying $v_{\mathrm{abs}}$ and $v_{\mathrm{p}}, \xi$ must be adjusted to compensate for its effect on the level of the observed flux and to ensure the inverse Compton spectra do not exceed the INTEGRAL upper limits, while minimising $\mathcal{D}$.

Figure 2 shows the measurements and variable ranges obtained from the simultaneous multi-frequency observation of S5 $0716+714$ from the study conducted by Ostorero et al. (2006). Also shown are the spectra predicted by the model assuming electrons are monoenergetic. The Doppler boosting factor is $\mathcal{D}=55$ in both models. The weak-magnetic-field model has a synchrotron self-absorption frequency of $v_{\mathrm{abs}}=3.9 \mathrm{GHz}$ with a synchrotron spectrum that peaks at $v_{\mathrm{p}}=300 \mathrm{GHz}$ (the values of other parameters are shown in Table 1). This model gives a brightness temperature of $T_{\mathrm{B}}=3.9 \times 10^{12} \mathrm{~K}$ at $v_{\mathrm{obs}}=$ $32 \mathrm{GHz}\left(T_{\mathrm{B}}=c^{2} F_{v} /\left(2 k_{\mathrm{B}} v^{2} \theta_{\mathrm{d}}^{2}\right)\right.$, where $k_{\mathrm{B}}$ is the Boltzmann constant and $\theta_{\mathrm{d}}$ the angular diameter of the source). The synchrotron spectrum shows good agreement with the data points at radio frequencies. The first-order inverse-Compton spectrum gives emission from optical to soft X-ray frequencies and the secondorder spectrum gives gamma-ray emission up to $\sim 40 \mathrm{MeV}$, while emission from higher orders scattering is negligible due to the Klein-Nishina effect.

The strong-magnetic-field model has $v_{\mathrm{abs}}=3.9 \mathrm{GHz}$, with its synchrotron peak at $v_{\mathrm{p}}=55 \times 10^{12} \mathrm{~Hz}$ (the values of other parameters can be found in Table 1) and gives a brightness temperature of $T_{\mathrm{B}}=3.7 \times 10^{12} \mathrm{~K}$ at the observing frequency $v_{\text {obs }}=32 \mathrm{GHz}$. The synchrotron spectrum extends up to optical frequencies, and gives a reasonable fit at radio frequencies up to $\sim 10^{11.5} \mathrm{~Hz}$. The first-order inverse-Compton spectrum gives X-ray emission, the second-order inverse-Compton spectrum is greatly reduced by the Klein-Nishina effects, and very little gamma-ray emission is produced.

The spectral break at $10^{11.5} \mathrm{~Hz}$ is well-fitted by the weakmagnetic-field model. We are unable to obtain a set of parameters to allow the first inverse Compton spectrum to reproduce the optical data. Simple qualitative analysis shows that mimicking the optical data points with the first inverse Compton spectrum is inconsistent with observation. The level of flux that the first inverse-Compton spectrum would require in order to account for the optical data is much higher than the synchrotron flux (i.e. $\xi \gg 1$ ), so a large $\gamma$ would therefore be required, resulting in the spectrum extending to frequencies far beyond the optical band. The first inverse-Compton spectrum would therefore exceed the INTEGRAL upper limits, and the very high X-and $\gamma$-ray flux would result in copious electron-positron pair production as the $\gamma$-ray photons interact with the synchrotron photons.

Alternatively, one can attempt to include the optical data in the synchrotron spectrum, as shown by the strong-magnetic-field model. The Lorentz factor of this model is higher than $\gamma_{\text {cool }}$, which implies that the particles lose a significant portion of their energy by synchrotron radiation before they vacate the source, so the electron spectrum will evolve into one that is proportional to $\gamma^{-2}$. This set of parameters therefore violates the monoenergetic assumption. Furthermore, the predicted spectrum fails to account for the spectral break at $v \sim 10^{11.5} \mathrm{~Hz}$, and the optical flux is very sensitively to the electron Lorentz factor. This model is, therefore, inconsistent. It is apparent that, in order to reproduce the observed optical emission, a power-law component in the electron spectrum at $\gamma>\gamma_{\mathrm{p}}$ must be incorporated, which emits synchrotron radiation at a frequency above $v_{\mathrm{p}}$.

\subsection{Injection of electrons in a double power law}

The Comptonisation parameter $\xi$ in this model must account for the $\gamma$ dependence of the electron density. It is therefore redefined as

$\xi=\frac{4}{3} R \sigma_{\mathrm{T}} \int_{0}^{\infty} \gamma^{2} n_{\mathrm{e}} \mathrm{d} \gamma$,

which is consistent with the definition used in the monoenergetic model. However, for the purpose of fitting the flux in the INTEGRAL frequency range, $\sim 10^{18}-10^{20} \mathrm{~Hz}$, we introduce a parameter $r_{\mathrm{p}}$, that determines the ratio of flux between $v_{\mathrm{p}}$ and $\gamma_{\mathrm{p}}^{2} v_{\mathrm{p}}$. Inspection of the solid line in Fig. 2 shows that synchrotron photons at the spectral break $v_{\mathrm{p}} \approx 300 \mathrm{GHz}$ are scattered to $\gamma_{\mathrm{p}}^{2} v_{\mathrm{p}} \approx 10^{17.5} \mathrm{~Hz}$. Therefore, $r_{\mathrm{p}}$ is a parameter that allows us to specify the spectral break $v_{\mathrm{p}}$ and then to adjust the flux in the INTEGRAL frequency range. The normalisation constant in the double power-law injection spectrum, $Q_{0}$ is eliminated in favour of the parameter $r_{\mathrm{p}}$

$r_{\mathrm{p}}=\frac{4}{3} \gamma_{\mathrm{p}}^{2} R \sigma_{\mathrm{T}} \int_{0}^{\infty} n_{\mathrm{e}}(\gamma) \mathrm{d} \gamma$,

with $n_{\mathrm{e}}(\gamma)$ given by Eq. (12). In the monoenergetic approximation, $r_{\mathrm{p}}$ is equivalent to $\xi$. Therefore, using the monoenergetic model and specifying $v_{\mathrm{abs}}$ and $v_{\mathrm{p}}$, the radio and the X-ray flux can be adjusted with $\xi \equiv r_{\mathrm{p}}$ and $\mathcal{D}$, and $v_{\text {cool }}$ can be calculated.

In the low-Doppler-factor model (Figs. 3 and 4), we attempt to minimise the Doppler factor of the source. According to Wagner et al. (1996) and Ostorero et al. (2006), the variability displayed by S5 $0716+714$ is intrinsic, and the variation time $\Delta t=4.1$ days was measured at $32 \mathrm{GHz}$ and $37 \mathrm{GHz}$. Therefore, we require that the model spectrum must agree with the data 


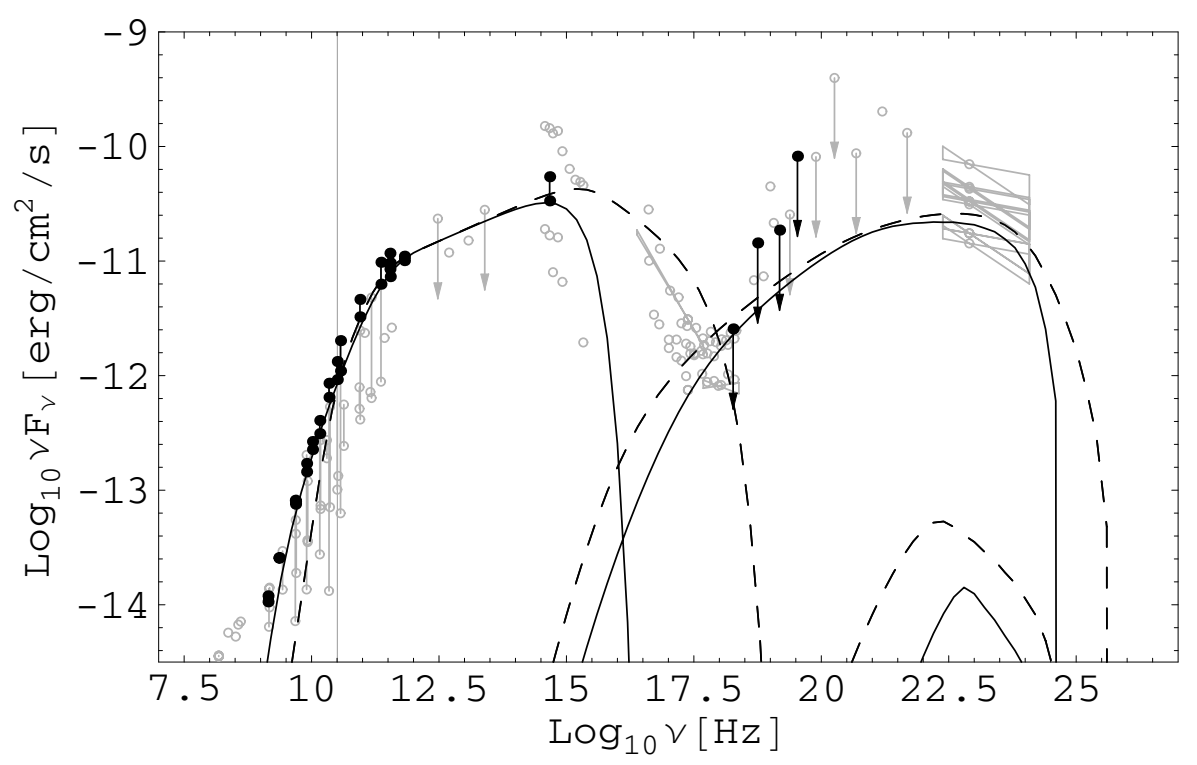

Fig. 3. The spectral energy distribution of S5 $0716+714$, as represented in Fig. 2. The model spectra, shown as solid and dashed lines, are computed from a quasi-monoenergetic electron distribution in the form of Eq. (12). The dashed line represents the low-Doppler-factor model where the Doppler boosting factor is minimised, whereas the solid line shows the high-Doppler-factor model where the values of the parameters are chosen to account for all radio and optical data points. The dashed gridline shows the position of $32 \mathrm{GHz}$. The values of the parameters are shown in Table 1. Historical data, as compiled by Ostorero et al. (2006) and shown as grey symbols, at radio-to-optical frequencies are from Kuehr et al. (1981), Waltman et al. (1981), Eckart et al. (1982), Perley (1982), Perley et al. (1982), Lawrence et al. (1985), Saikia et al. (1987), Kuehr \& Schmidt (1990), Moshir et al. (1990), Hales et al. (1991), Krichbaum et al. (1993), Gear et al. (1994), Hales et al. (1995), Douglas et al. (1996), Rengelink et al. (1997), Zhang et al. (1997), Riley et al. (1999), Cohen et al. (2002), Raiteri et al. (2003); UV data from Pian \& Treves (1993), Ghisellini et al. (1997); X-ray data from Biermann et al. (1992), Comastri et al. (1997), Kubo et al. (1998), Giommi et al. (1999), Tagliaferri et al. (2003), Pian et al. (2005); and $\gamma$-ray data from McNaron-Brown et al. (1995), Hartman et al. (1999), Collmar (2006).

at these two frequencies. We are unaware of correlated simultaneous variability measurement at lower frequencies during this campaign and, therefore, allow the model spectrum to deviate from the data at frequencies below $32 \mathrm{GHz}$. At the expense of having a lower flux than what is observed at frequencies below $32 \mathrm{GHz}$, we find that the minimum Doppler factor required is reduced to $\mathcal{D}=30$.

The power-law indices of the injection spectrum used to generate the spectrum of the low-Doppler-factor model are $s_{1}=-2$ for the low-energy part, such that electrons with $\gamma<\gamma_{\mathrm{p}}$ do not contribute significantly to the synchrotron opacity, and $s_{2}=$ 2.60, chosen for constructing the spectral shape in the infrared to optical band. The rest of the parameters are varied while keeping the Doppler boosting factor fixed. To find the limiting case, we have chosen the self absorption frequency to be $v_{\mathrm{abs}}=32 \mathrm{GHz}$ and find that the minimum Doppler factor that can generate a high enough level of flux at $32 \mathrm{GHz}$ and beyond is $\mathcal{D}=30$. The values of the other parameters can be found in Table 1. At the observing frequency of $32 \mathrm{GHz}$, the brightness temperature in the frame of the observer is $T_{\mathrm{B}}=1.4 \times 10^{13} \mathrm{~K}$. The frequency at which the synchrotron spectrum cuts off does not affect the spectral shape at low frequencies. However, $v_{\max }=\mathcal{D} /(1+z) \times$ $v_{0} \gamma_{\max }^{2}$ is constrained by the optical data, which imposes a lower limit on $v_{\max }$, and the INTEGRAL upper limits, which impose an upper limit on $v_{\max }$. The maximum value is shown in this model, where $v_{\max }=10^{18} \mathrm{~Hz}$. This equates to $\gamma_{\max }=5.45 \times 10^{5}$ with $\mathcal{D}=30$ and $z=0.3$.

The low-Doppler-factor model shows that it is possible to interpret the observed variability at $32 \mathrm{GHz}$ and $37 \mathrm{GHz}$ as coming from one of the jet components with the kinematics described by Bach et al. (2005). That would require the lower-frequency emission to originates from a larger region than what is inferred from the observed variability at $v \geq 32 \mathrm{GHz}$.

In the high-Doppler-factor model (Figs. 3 and 4) we assume the emission at all frequencies - including the low frequency $(<32 \mathrm{GHz})$ radio - originates from a single homogeneous source. This is suggested by the correlation between the variability at $5 \mathrm{GHz}$ and $650 \mathrm{~nm}$ observed in February 1990 (Quirrenbach et al. 1991; Wagner et al. 1996). The parameter $r_{\mathrm{p}}$ must be kept small, so that the inverse Compton spectra are below the INTEGRAL upper limits. This is achieved at the expense of a relatively high Doppler factor, at $\mathcal{D}=65$. The brightness temperature at $32 \mathrm{GHz}$ is $T_{\mathrm{B}}=2.5 \times 10^{12} \mathrm{~K}$. This model also shows the minimum value of $v_{\max }$, found to be $v_{\max }=1.5 \times 10^{15} \mathrm{~Hz}$.

The high-Doppler-factor model shows that if the emission at all frequencies originate from a single source region, it must be boosted by a much higher Doppler factor than proposed for the jet components by Bach et al. (2005). Even with a viewing angle $\vartheta \approx 0$, in which case $\mathcal{D} \approx 2 \Gamma$, a bulk Lorentz factor of $\Gamma>33$ is required, suggesting either that the source was travelling at a much higher speed than during the observations analysed by Bach et al. (2005) or that an interpretation of the superluminal motion in the VLBI jet that infers a much higher bulk Lorentz factor should be applied. One such suggestion is a conical jet (Gopal-Krishna et al. 2006). Alternatively, the jet components may decelerate as they travel down the jet (Marscher 1999; Georganopoulos \& Kazanas 2003; Ghisellini et al. 2005). The wide range of superluminal velocities shown in Bach et al. (2005) would then be a combined result of the variations in speed, as well as of the viewing angle. 

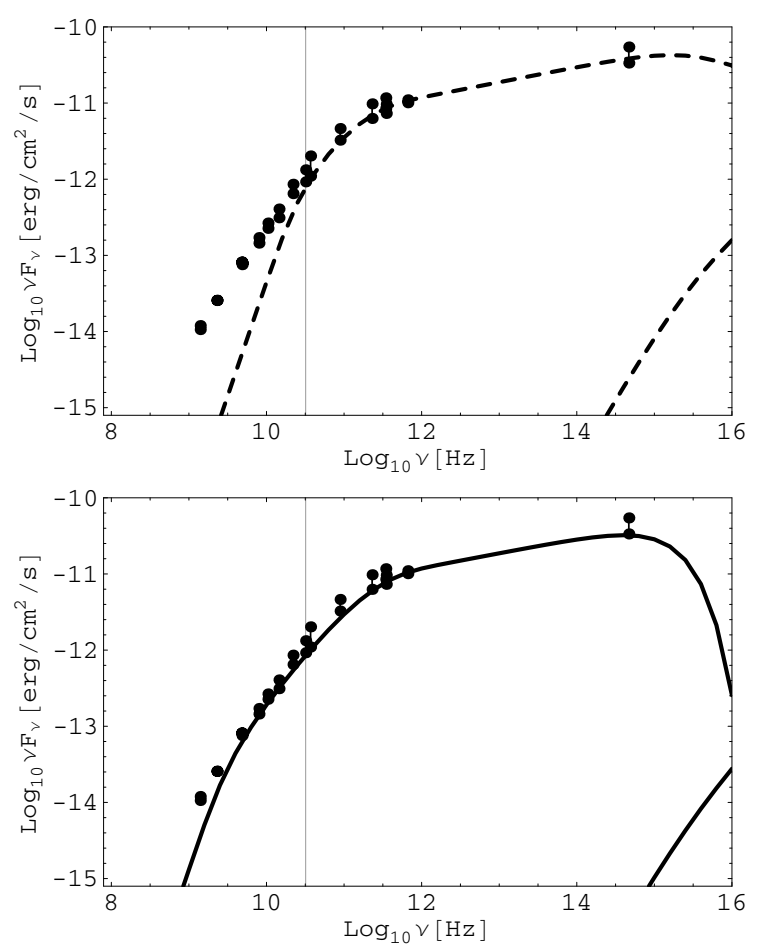

Fig. 4. The spectral energy distribution of S5 $0716+714$ and the model spectra, as represented in Fig. 2, in the radio-to-optical band. Top panel shows the low-Doppler-factor model where the Doppler boosting factor is minimised. Bottom panel shows the high-Doppler-factor model where the values of the parameters are chosen to account for all radio and optical data points.

\section{Discussion}

The spectra of the four models - two monoenergetic and two with double power-law electron injection - shown in Figs. 2 and 3, show brightness temperatures at $32 \mathrm{GHz}$ that are well above the conventional Compton limit. However, due to the lack of low-energy electrons, these brightness temperatures in fact lie below the threshold of the Compton catastrophe (i.e., $\xi<1$ ) and are, therefore, sustainable by the source.

An independent limit on $T_{\mathrm{B}}$ is provided by induced Compton scattering, when low-energy electrons couple with highfrequency photons. The photon occupation number $n_{\mathrm{ph}}(v) \propto$ $I_{v} / v^{3}$, which implies that the photon occupation number is high at and below the peak (where $v=v_{\text {abs }}$ ) of the synchrotron spectrum. In the presence of low-energy electrons, induced Compton scattering of photons at frequency $v_{\mathrm{abs}}$ to frequencies $v<v_{\mathrm{abs}}$ becomes increasingly significant as the synchrotron flux grows. Sunyaev (1971) showed that this process limits the brightness temperature at a certain frequency to $T_{\mathrm{B}}<m c^{2} /\left(k_{\mathrm{B}} \tau_{\mathrm{T}}\right)=5 \times$ $10^{9} \mathrm{~K}$ for $\tau_{\mathrm{T}} \sim 1$. Sincell \& Krolik (1994) demonstrated by numerical simulations that relativistic induced Compton scattering limits the brightness temperature of a self-absorbed synchrotron source to $T_{\mathrm{B}}<2 \times 10^{11} v_{\mathrm{GHz}}^{-1 /(s+3)} \gamma_{\min }^{(s+2) /(s+5)} \mathrm{K}$, where $v_{\mathrm{GHz}}$ is the observing frequency in units of $\mathrm{GHz}, \gamma_{\min }$ is the low energy cut-off in the electron spectrum $\propto \gamma^{-s}$. For a conventional powerlaw electron spectrum in which $\gamma_{\min }=1$, this gives a limit of $T_{\mathrm{B}}<2 \times 10^{11} \mathrm{~K}$ at $1 \mathrm{GHz}$.

One might suspect that, at such high brightness temperatures as are predicted by the models shown here, the effect of induced Compton scattering should be significant. Qualitative arguments reveal the contrary in our model, since the low-energy cut-off in the electron spectrum is effectively $\gamma_{\mathrm{p}}$, and the occupation number $\left(\propto F_{v} / v^{3}\right)$ of photons at frequencies that would couple with electrons at $\gamma_{\mathrm{p}}$ is negligibly small. Alternatively, when using the result of Sincell \& Krolik (1994), one finds that the limit corresponds to $T_{\mathrm{B}}<1.3 \times 10^{13} v_{\mathrm{GHz}}^{-2 / 11}\left(\gamma_{\mathrm{p}} / 10^{3}\right)^{3 / 5} \mathrm{~K}$ for $s=s_{2} \approx$ 2.6 and $\gamma_{\min }=\gamma_{\mathrm{p}}$.

The spectral break at $\sim 10^{11.5} \mathrm{~Hz}$ was interpreted by Ostorero et al. (2006) as the result of the change in opacity of the source. In our interpretation, the break is a result of a corresponding spectral break in the electron spectrum; below this frequency, the synchrotron spectrum remains optically thin. The self-absorption frequency $v_{\text {abs }}$ lies at a much lower frequency $(\sim 4 \mathrm{GHz})$ in our model, which implies a weaker magnetic field and/or a lower electron density. This interpretation therefore does not require an inhomogeneous magnetic field and electron density.

The spectral index of the high-energy "tail" of the synchrotron spectrum beyond the first spectral break depends on whether $\gamma_{\mathrm{p}}$ lies below or above $\gamma_{\mathrm{cool}}$, since this affects the final shape of the electron spectrum, as explained in Sect. 3. If the number of electrons leaving the energy $\gamma_{\mathrm{p}} m c^{2}$ is dominated by cooling by radiation, the synchrotron spectrum continues from $F_{v} \propto v^{1 / 3}$ between $v_{\text {abs }}$ and $v_{\text {cool }}$, to $F_{v} \propto v^{-1 / 2}$ between $v_{\text {cool }}$ and $v_{\mathrm{p}}$, then $F_{v} \propto v^{-s_{2} / 2}$ between $v_{\mathrm{p}}$ and $v_{\text {max }}$, and it is cut off exponentially beyond $v_{\max }$. In this case, the low radio-frequency spectrum resembles that of a monoenergetic electron distribution of energy $\gamma_{\mathrm{cool}} m c^{2}$. If, on the other hand, losses are dominated by electrons evacuating the emission zone on a time scale of $t_{\mathrm{esc}}=R / c$, the synchrotron spectrum then continues from $F_{v} \propto v^{1 / 3}$ between $v_{\text {abs }}$ and $v_{\mathrm{p}}$, to $F_{v} \propto v^{-\left(s_{2}-1\right) / 2}$ between $v_{\mathrm{p}}$ and $v_{\text {cool }}$, then $F_{v} \propto v^{-s_{2} / 2}$ between $v_{\text {cool }}$ and $v_{\text {max }}$, and it is again cuts off exponentially beyond $v_{\max }$. The low radio-frequency synchrotron spectrum can be approximated in the same way as the one from a monoenergetic distribution of electron of energy $\gamma_{\mathrm{p}} m c^{2}$.

Observations of S5 $0716+714$ from infrared to optical frequencies suggest that the spectral energy distribution between the frequencies $v_{\mathrm{K}}=1.38 \times 10^{14} \mathrm{~Hz}$ and $v_{\mathrm{B}}=6.81 \times 10^{14} \mathrm{~Hz}$ can be well-fitted by the power law $F_{v} \propto v^{-1.12}$ (Hagen-Thorn et al. 2006). Clearly, the top panel in Fig. 4 is much too hard at these frequencies. However, the spectrum can be softened by lowering the cut-off frequency of the synchrotron spectrum $v_{\max }$ (i.e., bottom panel in Fig. 4). By decreasing $v_{\max }$ to approximately $v_{\mathrm{K}}$, the spectrum begins an exponential drop at or just before reaching the relevant frequency range and, as a result, softens the spectrum, without altering the level of flux or the spectral shape at frequencies $<\nu_{\max }$.

Figure 2 and the bottom panel in Fig. 4 demonstrate that, if the variability of S5 $0716+714$ is intrinsic and extends down to $<32 \mathrm{GHz}$, the Doppler-boosting factor of the emission region must be much higher than the 20-30 suggested by Bach et al. (2005). One possibility for enabling the Doppler factor to fall within this range is to increase the magnetic field strength and the electron density, as shown in Table 1 . This causes the synchrotron self-absorption frequency $v_{\text {abs }}$ to increase, as shown by the top panel in Fig. 4, therefore requiring the assumption that the emission at frequency below $v_{\text {abs }}$ originates from a larger region than inferred from the variability at $32 \mathrm{GHz}$ and $37 \mathrm{GHz}$. Requiring the value of $v_{\mathrm{abs}} \leq 32 \mathrm{GHz}$, we find that, to remain below the INTEGRAL upper limits, we require a minimum Doppler factor of $\mathcal{D}=30$ and a minimum self-absorption frequency of $v_{\mathrm{abs}}=32 \mathrm{GHz}$. Below these minima, the model spectrum would either have a level of flux below the measured 

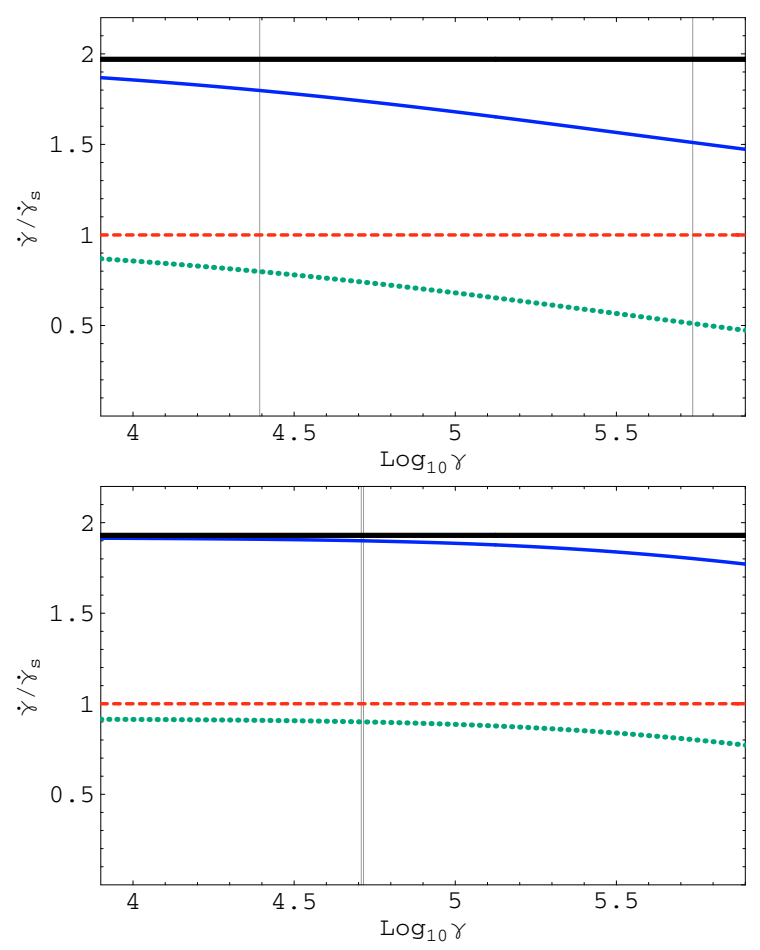

Fig. 5. Cooling rates normalised to the synchrotron loss rates (dashed lines at $\dot{\gamma}=\dot{\gamma}_{\mathrm{s}}$ ). The rate for inverse Compton scattering is shown as a dotted line, the total loss rate as a thin solid line (blue online). The approximate rate used to compute the electron distribution is shown as a thick solid line (black online). The top (bottom) panel shows the results of the low(high)-Doppler-factor model shown as dashed (solid) lines in Fig. 3. The vertical dotted lines correspond to $\gamma=a \gamma_{\text {cool }}$ (see Eq. (12)) on the left, and to $\gamma=\gamma_{\max }$ on the right.

flux at radio frequencies or the subsequent first inverse Compton spectrum would lie above the INTEGRAL upper limits.

The models shown here depart from the equipartition of magnetic and particle energy, and are dominated by the energy of the relativistic electrons (except for the rejected model). Estimating the energy required by the source from the host galaxy, $P_{\text {jet }}$, we find that the power required from the source by our models is consistent with what is expected from a lowenergy peaked BL Lac object (see e.g., Nieppola et al. 2006).

An approximation inherent in our method is that, as far as their effect on the electron distribution is concerned, inverse Compton losses are dominated by single scatterings off the synchrotron photons and may be treated in the Thomson approximation. For each of the models presented in Fig. 4, we show in Fig. 5 the total rate of radiative cooling of an electron (thin solid lines, blue online) computed using the full Klein-Nishina loss rate for scattering on the full output spectrum. This should be compared with the thick solid lines (black online) that give the loss rates used in computing the electron density according to Eq. (3). These lines are horizontal and give the quantity $\dot{\gamma}_{\text {total }} / \dot{\gamma}_{\mathrm{s}}=1+\delta$ for the converged model solutions. The range over which cooling is important in our approximate solution lies between the vertical lines at $\gamma=a \gamma_{\text {cool }}$ and $\gamma_{\max }$ in this figure. (In the lower panel, these lines lie close together.) The maximum deviation in this range is roughly $20 \%$ and occurs at $\gamma=\gamma_{\max }$ for the model with a relatively low Doppler factor (plotted as a dashed line in Fig. 4). The deviation for the higher Doppler factor model is less than $3 \%$. We conclude that this approximation has a negligible effect on the electron distribution. The computation of the output spectrum from the electron distribution is not affected by this approximation, since the full Klein-Nishina expression for the emissivity is used.

A testable prediction of our models is correlated variability. The hard $\alpha \approx-0.3$ radio spectral component between $30 \mathrm{GHz}$ and several hundred $\mathrm{GHz}$ is interpreted as the optically thin emission of electrons whose characteristic synchrotron frequency lies at or above hundreds of GHz. Consequently, fluctuations in the number of such particles should be simultaneously reflected in broad-band fluctuations of the specific intensity of radiation at these frequencies. We are not aware of studies that investigate such an effect.

The variability of the synchrotron spectrum should also be correlated to the variability of the inverse Compton spectrum, since the same electrons are responsible for both emissions. Analysis of XMM observation by Ferrero et al. (2006) show correlated variability between two X-ray spectral components of S5 0716+714, which were interpreted as the high-energy part of the synchrotron spectrum and the low-energy part of the inverse Compton spectrum. In the context of our model, the synchrotron component is the emission from the high-energy tail of the electron spectrum, whereas the IC component is the synchrotron emission of the hard low-energy electron spectrum scattered by the same low energy electrons. The study by Ferrero et al. (2006) therefore does not provide a direct test of our model, although their data support an SSC interpretation.

Similarly, the polarisation properties of the $30-100 \mathrm{GHz}$ emission are predicted to be those of the single-particle synchrotron emission. One aspect of this is the possibility that the intrinsic circular polarisation of the source might reach a few percent (Kirk \& Tsang 2006). Another concerns the degree of linear polarisation. Power-law electron distributions radiating in a source with a completely homogeneous magnetic field can theoretically reach quite high degrees of linear polarisation (up to $\approx 70 \%$, depending weakly on the power-law index). These values are not reached in extra-galactic radio sources, probably because the magnetic field direction within the source is tangled. In general, higher degrees of polarisation are found at higher frequency, consistent with the conventional picture in which the source size decreases with increasing frequency.

In our model, however, the degree of linear polarisation in the radio is determined by monoenergetic electrons. According to standard synchrotron theory, this tends to $50 \%$ at low frequency $\left(x=v / v_{\mathrm{S}} \ll 1\right)$, rises to $76 \%$ at $x=1$, and tends to $100 \%$ at $x \gg 1$. The effect of a tangled field within the source reduces these values, but is independent of observing frequency. Thus, as in conventional inhomogeneous models, the degree of linear polarisation is predicted to increase with frequency. However, more quantitative predictions would require consideration of effects, such as internal Faraday rotation, and lie outside the scope of the present paper.

Finally, we note that the hard electron injection spectrum that we have adopted $\left(n_{\mathrm{e}} \propto \gamma^{2}\right)$ corresponds to the lowenergy $\left(\gamma m c^{2}<k_{\mathrm{B}} T\right)$ part of a relativistic thermal distribution. According to Table 1, the corresponding electron temperature would lie at around $10^{12} \mathrm{~K}$. In an electron-ion plasma, this corresponds to the temperature of shocked gas behind a mildly relativistic shock front, if one assumes that the electrons and ions equilibrate to a common temperature. Recent P.I.C. simulations suggest that this assumption may indeed be justified (Spitkovsky 2007). 


\section{Conclusion}

Using the specific case of S5 $0716+714$ as an example, we confirm that it is possible to produce high brightness temperatures at $\mathrm{GHz}$ frequencies in compact radio sources without the onset of catastrophic cooling, provided that the radiating particles have a distribution that is sufficiently hard below a characteristic. In addition, we show qualitatively that induced Compton scattering is insignificant in sources with a low-energy electron cut-off despite the high brightness temperature, the underlying reason being the low occupation number of the photons that can couple with the electrons at the cut-off energy.

The model where an electron distribution that is a double power law in energy, peaking at $\gamma_{\mathrm{p}}$, is injected into the source offers more flexibility at higher frequencies in the synchrotron spectrum (from infrared to optical) at the expense of more free parameters, compared to either monoenergetic or single powerlaw distributions. These parameters should be constrained by simultaneous observations due to the highly variable nature of IDV sources. In the case of S5 $0716+714$ where such data is available, the spectral break at about $230 \mathrm{GHz}$ determines the value of $\gamma_{\mathrm{p}}$, the optical data at $5 \times 10^{14} \mathrm{~Hz}$ gives the lower limit of $v_{\max }$, as well as constraining the spectral index $s_{2}$, and the INTEGRAL upper limits give the upper limit of $v_{\max }$ and also constrain the value of $r_{\mathrm{p}}$, which in turn determines the electron density.

The example of S5 0716+714 illustrates several important spectral properties of an electron distribution with a low-energy cut-off, as described in the previous sections. The most noticeable feature is the hard, inverted, optically thin synchrotron spectrum, spanning a wide frequency range, which is a prevalent feature in compact radio sources at radio frequencies (e.g., Gear et al. 1994; Kedziora-Chudczer et al. 2001). Other features are the spectral breaks at $v_{\mathrm{p}}=\gamma_{\mathrm{p}}^{2} v_{0}, v_{\text {cool }}=\gamma_{\text {cool }}^{2} v_{0}$, and the exponential cut-off at $v_{\max }=\gamma_{\max }^{2} v_{0}$. This model, therefore, allows a simple homogeneous source to reproduce the common features shown by many IDV sources.

Acknowledgements. We thank Luisa Ostorero and Stefan Wagner for helpful discussions and for providing us with easy access to the observational data. We would also like to thank the anonymous referee for constructive comments and suggestions that we feel have led to a significant improvement in this paper.

\section{References}

Agudo, I., Krichbaum, T. P., Ungerechts, H., et al. 2006, A\&A, 456, 117

Bach, U., Krichbaum, T. P., Ros, E., et al. 2005, A\&A, 433, 815

Biermann, P. L., Schaaf, R., Pietsch, W., et al. 1992, A\&AS, 96, 339

Blandford, R. D., \& Konigl, A. 1979, ApJ, 232, 34

Bychkova, V. S., Kardashev, N. S., Boldycheva, A. V., Gnedin, Y. N., \& Maslennikov, K. L. 2006, Astron. Rep., 50, 802

Cohen, A. S., Lane, W. M., Cotton, W. D., et al. 2002, in BAAS, 1274

Collmar, W. 2006, in Blazar Variability Workshop II: Entering the GLAST Era, ed. H. R. Miller, K. Marshall, J. R. Webb, \& M. F. Aller, ASP Conf. Ser., 350, 120

Comastri, A., Fossati, G., Ghisellini, G., \& Molendi, S. 1997, ApJ, 480, 534 de Bruyn, A. G. 1976, A\&A, 52, 439

Douglas, J. N., Bash, F. N., Bozyan, F. A., Torrence, G. W., \& Wolfe, C. 1996, AJ, 111, 1945

Eckart, A., Pauliny-Toth, I. I. K., Witzel, A., et al. 1982, A\&A, 108, 157

Ferrero, E., Wagner, S. J., Emmanoulopoulos, D., \& Ostorero, L. 2006, A\&A, 457, 133

Foschini, L., Tagliaferri, G., Pian, E., et al. 2006, A\&A, 455, 871

Gear, W. K., Stevens, J. A., Hughes, D. H., et al. 1994, MNRAS, 267, 167

Georganopoulos, M., \& Kazanas, D. 2003, ApJ, 594, L27

Georganopoulos, M., Kirk, J. G., \& Mastichiadis, A. 2001, ApJ, 561, 111

Ghisellini, G., Villata, M., Raiteri, C. M., et al. 1997, A\&A, 327, 61

Ghisellini, G., Tavecchio, F., \& Chiaberge, M. 2005, A\&A, 432, 401

Giommi, P., Massaro, E., Chiappetti, L., et al. 1999, A\&A, 351, 59

Gopal-Krishna, Wiita, P. J., \& Dhurde, S. 2006, MNRAS, 369, 1287

Hagen-Thorn, V. A., Larionov, V. M., Efimova, N. V., et al. 2006, Astron. Rep., 50,458

Hales, S. E. G., Mayer, C. J., Warner, P. J., \& Baldwin, J. E. 1991, MNRAS, 251, 46

Hales, S. E. G., Waldram, E. M., Rees, N., \& Warner, P. J. 1995, MNRAS, 274, 447

Hartman, R. C., Bertsch, D. L., Bloom, S. D., et al. 1999, ApJS, 123, 79

Kardashev, N. S. 1962, Sov. Astron., 6, 317

Kedziora-Chudczer, L. L., Jauncey, D. L., Wieringa, M. H., Tzioumis, A. K., \& Reynolds, J. E. 2001, MNRAS, 325, 1411

Kellermann, K. I. 2002, Publ. Astron. Soc. Austr., 19, 77

Kellermann, K. I., \& Pauliny-Toth, I. I. K. 1969, ApJ, 155, L71

Kirk, J. G., \& Tsang, O. 2006, A\&A, 447, L13

Krichbaum, T. P., Witzel, A., Graham, D. A., et al. 1993, A\&A, 275, 375

Kubo, H., Takahashi, T., Madejski, G., et al. 1998, ApJ, 504, 693

Kuehr, H., \& Schmidt, G. D. 1990, AJ, 99, 1

Kuehr, H., Witzel, A., Pauliny-Toth, I. I. K., \& Nauber, U. 1981, A\&AS, 45, 367

Lawrence, C. R., Readhead, A. C. S., Linfield, R. P., et al. 1985, ApJ, 296, 458

Longair, M. S. 1992, High energy astrophysics, Vol. 1,2: Particles, photons and their detection (Cambridge: Cambridge University Press), 2nd edn.

Macquart, J.-P., Kedziora-Chudczer, L., Rayner, D. P., \& Jauncey, D. L. 2000, ApJ, 538, 623

Marscher, A. P. 1977, ApJ, 216, 244

Marscher, A. P. 1999, Astropart. Phys., 11, 19

McNaron-Brown, K., Johnson, W. N., Jung, G. V., et al. 1995, ApJ, 451, 575

Moshir, M., Kopan, G., Conrow, T., et al. 1990, in BAAS, 1325

Nieppola, E., Tornikoski, M., \& Valtaoja, E. 2006, A\&A, 445, 441

Ostorero, L., Wagner, S. J., Gracia, J., et al. 2006, A\&A, 451, 797

Perley, R. A. 1982, AJ, 87, 859

Perley, R. A., Fomalont, E. B., \& Johnston, K. J. 1982, ApJ, 255, L93

Pian, E., \& Treves, A. 1993, ApJ, 416, 130

Pian, E., Foschini, L., Beckmann, V., et al. 2005, A\&A, 429, 427

Quirrenbach, A., Witzel, A., Wagner, S., et al. 1991, ApJ, 372, L71

Raiteri, C. M., Villata, M., Tosti, G., et al. 2003, A\&A, 402, 151

Readhead, A. C. S. 1994, ApJ, 426, 51

Rengelink, R. B., Tang, Y., de Bruyn, A. G., et al. 1997, A\&AS, 124, 259

Rickett, B. J., Kedziora-Chudczer, L., \& Jauncey, D. L. 2002, ApJ, 581, 103

Riley, J. M. W., Waldram, E. M., \& Riley, J. M. 1999, MNRAS, 306, 31

Rybicki, G. B., \& Lightman, A. P. 1979, Radiative processes in astrophysics (New York: Wiley-Interscience), 393

Saikia, D. J., Salter, C. J., Neff, S. G., et al. 1987, MNRAS, 228, 203

Sincell, M. W., \& Krolik, J. H. 1994, ApJ, 430, 550

Sokolov, A., \& Marscher, A. P. 2005, ApJ, 629, 52

Spitkovsky, A. 2007, ArXiv e-prints, 706

Sunyaev, R. A. 1971, Sov. Astron., 15, 190

Tagliaferri, G., Ravasio, M., Ghisellini, G., et al. 2003, A\&A, 400, 477

Tsang, O., \& Kirk, J. G. 2007, A\&A, 463, 145

Wagner, S. J. 2001, Ap\&SS, 278, 105

Wagner, S. J., \& Witzel, A. 1995, ARA\&A, 33, 163

Wagner, S. J., Witzel, A., Heidt, J., et al. 1996, AJ, 111, 2187

Waltman, E., Johnston, K. J., Spencer, J. H., et al. 1981, A\&A, 101, 49

Zhang, X., Zheng, Y., Chen, H., et al. 1997, A\&AS, 121, 59 